\title{
Angine bulleuse hémorragique après des soins dentaires : présentation d'un cas
}

\author{
Christian Milin ${ }^{1, *}$, Benoit Fouche ${ }^{2}$ \\ 1 Service d'Odontologie, Centre hospitalier Camille Guérin, Châtellerault, France \\ 2 Pratique privée
}

(Reçu le 23 juin 2011, accepté le 12 septembre 2011)

\begin{abstract}
Mots clés :
angine bulleuse

hémorragique / bulle /

ulcération buccale

Résumé - L'angine bulleuse hémorragique constitue une affection bulleuse bénigne et méconnue de la cavité buccale et de l'oropharynx. Après une durée de vie limitée, la bulle hémorragique laisse place à une ulcération non spécifique qui peut constituer une réelle difficulté diagnostique. L'histologie est peu contributive et l'immunofluorescence négative. Le diagnostic est principalement clinique. Son étiologie et sa son pathogénie restent largement incomprises. Le seul facteur favorisant connu concerne l'utilisation d'aérosols de corticoïdes au long cours. L'évolution est le plus souvent spontanément favorable mais un cas exceptionnel d'obstruction des voies aériennes supérieures a été décrit. Des récidives apparaissent fréquemment. Un cas survenu chez une femme de 66 ans après des soins dentaires est rapporté.
\end{abstract}

Key words: angina

Abstract - Angina bullosa haemorrhagica after dental treatment: report of a case. Angina bullosa bullosa haemorrhagica / haemorrhagica is a benign and unrecognised affection of the oral cavity and oropharynx. The limited length life of blister / oral ulceration the blister make place for a non specific ulceration, which make up the main difficult diagnosis. Histology remains few contributive and immunofluorescence negative. Based on clinical diagnosis, its aetiology and pathogenesis remains widely unknown. The only known favorising factor is the use of long term inhalation of steroid. This evolution is spontaneously favourable, despite a exceptional case of upper airway obstruction. Recurrences appears frequently. A case in a 66 year-old woman after dental treatment is reported.

Rarement diagnostiquée, probablement sous-estimée en raison de son caractère fugace et peu symptomatique, l'angine bulleuse hémorragique constitue une affection bulleuse de la cavité buccale rare et étonnante.

Les auteurs présentent un cas siégeant sur le bord de la langue, apparu après des soins dentaires. Ils discutent l'aspect clinique, le diagnostic différentiel et l'attitude thérapeutique à adopter.

\section{Observation}

En janvier 2010, une patiente de 66 ans est adressée par son médecin traitant pour une douleur linguale récidivante. $A$ la première consultation, l'examen clinique est normal et ne montre aucune lésion buccale. La patiente décrit un épisode algique d'apparition brutale, suivi d'un saignement de la langue. Les symptômes cliniques évoluent favorablement et spontanément en 4 à 6 jours. L'anamnèse révèle l'existence d'une rectocolite hémorragique et d'une angine de poitrine traitées respectivement par mésalazine (Pentasa ${ }^{\circledR}$ ) et métoprolol (Lopressor ${ }^{\circledR}$ ), et un terrain atopique avec polyallergie (iode, pénicilline, clindamycine, spiramycine). Devant ce tableau clinique très fruste, les douleurs sont attribuées à des ulcérations aphtoïdes en rapport avec la rectocolite hémorragique [1].

Six mois après la première consultation, la patiente fait réaliser des soins dentaires conservateurs. Au cours d'une séance de soins, on observe l'apparition d'une bulle à contenu hémorragique, sur le bord latéral droit de la langue. Il s'agit d'une bulle tendue, indolore, unique (Fig. 1). La patiente est examinée deux jours plus tard dans un cabinet spécialisé en chirurgie et médecine buccales.

\footnotetext{
*Correspondance : christian.milin@orange.fr
} 


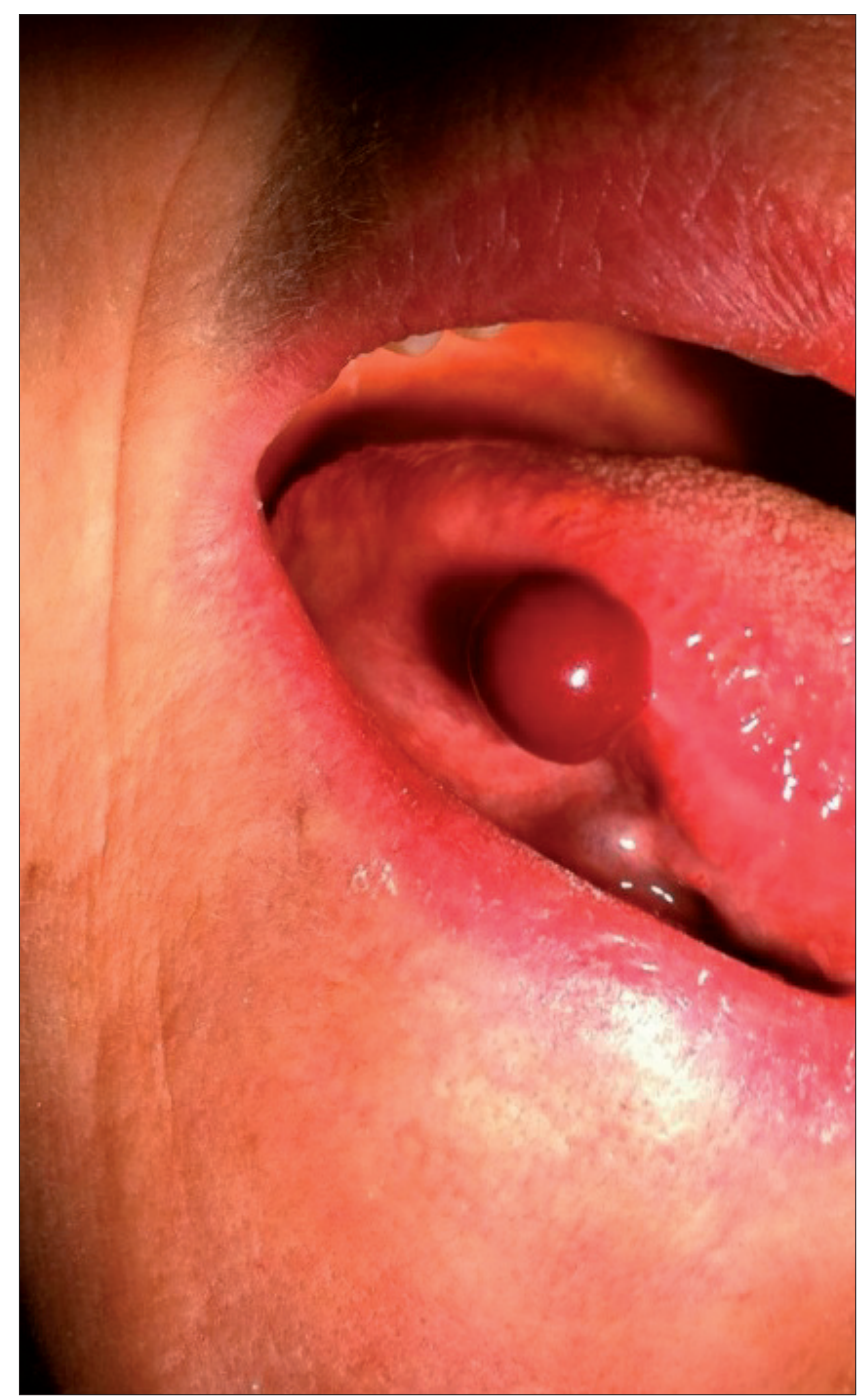

Fig. 1. Bulle hémorragique sur le bord droit de la langue. Fig. 1. Lingual haemorrhagic blister.

Devant ce tableau pathognomonique, le diagnostic d'angine bulleuse hémorragique a été posé. Aucun examen complémentaire n'a été réalisé ; le traitement a comporté la prescription d'antalgiques per os et d'antiseptiques locaux. La nature de cette affection a été expliquée à la patiente afin de la rassurer.

\section{Commentaires}

L'angine bulleuse hémorragique est l'une maladie bulleuse de la muqueuse buccale, peu décrite dans la littérature. La première description est habituellement attribuée à Badham en 1967 [2] ; Grinspan et al. considèrent qu'elle aurait déjà été faite par Balina en 1933 [3]. Cette affection se traduit par

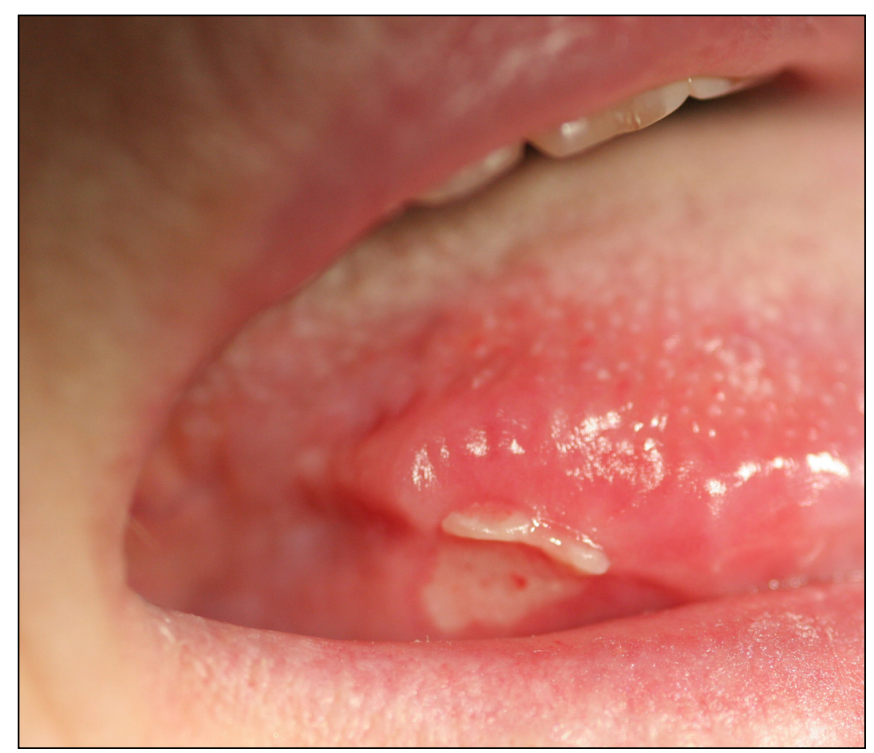

Fig. 2. Ulcération non spécifique $(J+2)$. Fig. 2. Non specific ulcération $(D+2)$.

le développement d'une bulle hémorragique récurrente sur la muqueuse buccale ou oropharyngée, sans être associée é une pathologie systémique $[2,4]$. L'apparition des lésions est parfois précédée d'une sensation de brûlures $[3,5,6]$. La bulle de couleur rouge ou violacée, se rompt spontanément après quelques heures, libérant son contenu hémorragique et laissant place à une ulcération non spécifique (Fig. 2), douloureuse qui guérit spontanément en quelques jours [7]. Certains patients décrivent un saignement prolongé [6]. La rupture d'une bulle oropharyngée peut s'accompagner d'une sensation d'étouffement [8].

L'angine bulleuse hémorragique intéresse principalement la muqueuse buccale, le plus souvent le palais mou et le palais dur, plus rarement la langue ou la face interne des lèvres, parfois dans la région oropharyngée. Dans cette localisation, une bulle volumineuse peut induire une situation de détresse respiratoire aiguë par obstruction des VADS [9]. Dans certains cas, la formation de la bulle semble favorisée par une intubation ou une extubation $[4,10]$.

L'incidence de cette affection est évaluée à 0,5\% [3]. Elle se manifeste le plus souvent à un âge intermédiaire ou avancé, avec une légère prédominance féminine (Tab. I) $[4,6,8,11,12]$. Une composante génétique a été suspectée [4]. Le cas le plus précoce a été rapporté par Giuliani et al. chez une adolescente de 13 ans [13].

Des affections systémiques sont parfois associées - lupus érythémateux disséminé [9], hypertension artérielle [12,14], thalassémie [13], diabète $[3,12]$, insuffisance rénale chronique [12], rectocolite hémorragique - mais aucune relation causale n'a pu être établie. 
Tableau I. Publications rapportant au moins trois cas d'angine bulleuse hémorragique.

Table I. Publications with more three cases of angina bullosa haemorrhagica.

\begin{tabular}{|l|c|c|c|}
\hline Auteurs & Nombre & $\%$ femmes & Age moyen (extrêmes) \\
\hline Hopkins et Walter (1985) & 9 & $67 \%$ & $40(22-70)$ \\
\hline Stephenson et al. (1987) & 30 & $53 \%$ & $54(20-83)$ \\
\hline Edwards et al. (1990) & 3 & $100 \%$ & $43(28-60)$ \\
\hline Deblauwe et Van der Wall (1994) & 9 & $67 \%$ & $63(42-84)$ \\
\hline Guiliani et al. (2002) & 8 & $50 \%$ & $46(13-70)$ \\
\hline Yamamoto et al. (2006) & 11 & $72 \%$ & $55(17-81)$ \\
\hline Horie et Kawana (2008) & 16 & $37 \%$ & $51(26-74)$ \\
\hline
\end{tabular}

Tableau II. Résumé de l'étude de High et Main [18] : rapport entre la durée de la corticothérapie par inhalation et la fréquence de l'angine bulleuse hémorragique.

Table II. Summary of the High and Main's study [18]: relation between long-term of steroid treatment and incidence of angina bullosa heamorrhagica.

\begin{tabular}{|l|c|c|c|}
\hline $\begin{array}{c}\text { Durée du } \\
\text { traitement }\end{array}$ & $\begin{array}{c}\text { Nombrede } \\
\text { patients }\end{array}$ & $\begin{array}{c}\text { Nombrede } \\
\text { cas }\end{array}$ & Pourcentage \\
\hline$<3$ ans & 22 & - & $0 \%$ \\
\hline 3 à 5 ans & 6 & 1 & $17 \%$ \\
\hline$>5$ ans & 14 & 10 & $71 \%$ \\
\hline
\end{tabular}

\section{Etiologie}

Elle n'est pas encore élucidée mais cette affection a probablement une origine multifactorielle. De par sa définition [2], aucun trouble de l'hémostase n'a jamais été mis en évidence au cours des épisodes où la bulle se développe $[4,8,9,13]$. Dans de nombreux cas, l'apparition des bulles a pu être corrélée à des traumatismes locaux lors de l'alimentation, de soins dentaires, de traitements parodontaux ou dus à des prothèses dentaires iatrogènes $[4,7,11,12,14-17]$. Un traumatisme initial est retrouvé dans $53 \%$ des cas [4] ; pour les autres cas, l'apparition semble spontanée. Un seul facteur favorisant a pu être identifié : le traitement au long cours (5 ans minimum) par des aérosols à base de corticoïdes favorisent le développement de bulles hémorragiques (Tab. II) $[8,18,19]$.

Aucun facteur systémique n'a pu être clairement identifié. Horie et al. [14], sans pouvoir établir de relation pathogénique, retrouve une prédominance de patients hypertendus dans un groupe de 16 patients; Grinspan et al. [3] 44\% de patients diabétiques.

\section{Pathogénie}

Dans la plupart des cas, la pathogénie reste inconnue $[5,13]$. Pour les patients sous corticoïdes au long cours, on retrouve des modifications de la quantité et de la qualité des fibres de collagéne et d'élastine, ce qui diminuerait l'ancrage de la muqueuse et fragiliserait les vaisseaux sanguins [8] ; ces modifications favorisant le développement de bulles hémorragiques.

\section{Anatomopathologie}

Elle est peu documentée. Badham [2] décrit des bulles intra-épithéliales, d'autres des bulles sous-épithéliales [4,11], voire des bulles dans le chorion superficiel [8]. La lésion est caractérisée dans sa phase d'état par une cavité remplie de sang [4]. Lorsque la bulle est rompue, on observe une ulcération non spécifique (Fig. 2), entourée par un épithélium d'aspect normal [13]. Un infiltrat constitué de polynucléaires peut être observé dans le chorion [7] ainsi qu'une absence quasi totale de fibres élastiques [18].

L'examen anatomopathologique et l'examen en immunofluorescence directe sont rarement effectués car ils se révèlent peu contributifs $[3,4,5,9,20]$.

\section{Diagnostic}

Bon nombre de cas ne sont probablement pas diagostiqués en raison de la fugacité des symptômes $[5,6,12]$, et le diagnostic est souvent posé après plusieurs années d'évolution $[5,7,8]$. Il est basé sur l'examen clinique : il est facile lorsque le praticien peut observer l'aspect pathognomonique (la bulle hémorragique), beaucoup plus incertain au stade d'ulcération non spécifique. Le signe de Nikolsky est toujours négatif $[5,9]$.

Le bilan biologique, indiqué seulement lorsqu'on suspecte un trouble de l'hémostase, ne révèle aucune anomalie $[2,4,9,14]$.

\section{Diagnostic différentiel}

Le tableau clinique fait évoquer une maladie bulleuse : avant tout le pemphigus, la pemphigoïde cicatricielle et 
l'érythéme polymorphe. Dans les deux premières affections, les bulles sont plus petites, multiples et rarement hémorragiques. En cas de doute, il faut réaliser un examen en immunofluorescence. Certaines formes d'érythème polymorphe ne comportent que des lésions buccales, quelquefois uniques, mais l'aspect anatomopathologique de ces lésions est bien différent (nécrose de l'épithélium). Dans les autres maladies bulleuses, les lésions sont de plus petites et toujours associées à des lésions cutanées. Des troubles de l'hémostase acquis ou méconnus (thrombopénie, maladie de Von Willebrand...) doivent être aussi évoqués [13] ; le bilan biologique oriente rapidement le diagnostic.

La persistance de l'ulcération au-delà d'une dizaine de jours doit faire envisager un autre diagnostic et réaliser une biopsie de la lésion.

\section{Traitement}

Il n'y a pas de traitement spécifique de l'angine bulleuse hémorragique. Il faut souvent d'abord rassurer le patient, puis prescrire éventuellement un traitement symptomatique (pâte antalgique - Dynexan ${ }^{\circledR}$, Xylocaine visqueuse ${ }^{\circledR}$ - à appliquer sur la zone ulcérée), avec des bains de bouche antiseptiques (chlorexidine) [4] afin d'éviter une éventuelle surinfection. Certains préconisent un traitement à base d'acide ascorbique $\left(200 \mathrm{mg} \times 2 \mathrm{j}^{-1}\right)$ afin de réduire le risque de récidive [3].

Pour les patients sous inhalation de corticoïdes, il faut rappeler la nécessité d'un rinçage systématique de la cavité buccale à l'eau immédiatement après la pulvérisation de l'aérosol ; ceci permettrait de réduire l'incidence des angines bulleuses hémorragiques [18].

Conflits d'intérêt : aucun

\section{Références}

1. Kuffer R, Lombardi T, Husson-Bui C, Courrier B, Samson J. La muqueuse buccale: de la clinique au traitement. Paris: Med'Com, 2009, 121 p.

2. Badham NJ. Blood blisters and oesophageal cast. J Laryngol 1967;81:791-803.

3. Grinspan D, Abulafia J, Lanfranchi H. Angina bullosa hemorrhagica. Int J Dermatol 1999;38:525-8.

4. Stephenson P, Scully C, Prime SS, Daly HM. Angina bullosa haemorrhagica: lesional immunostaining and haematological findings. Br J Oral Maxillofac Surg1987;25:488-91.
5. Higgins EM, du Vivier AW. Angina bullosa haemorrhagica - a possible relation to steroid inhalers. Clin Exp Dermatol 1991;16:244-6.

6. Deblauwe BM, van der Waal I. Blood blisters of the oral mucosa (angina bullosa haemorrhagica). J Am Acad Dermatol 1994;31:341-4.

7. De las Heras ME, Moreno R, Núñez M, Gómez MI, Ledo A. Angina bullosa hemorrhagica. J Dermatol 1996;23:507-9.

8. Edwards S, Wilkinson JD, Wojnarowska F. Angina bullosa haemorrhagica - a report of three cases and review of the literature. Clin Exp Dermatol 1990;15:422-4.

9. Pahl C, Yarrow S, Steventon N, Saeed NR, Dyar 0. Angina bullosa haemorrhagica presenting as acute upper airway obstruction. $\mathrm{Br}$ J Anaesth 2004;92:283-6.

10. Hosain SI, Bounds G, Stanford J. Angina haemorrhagica bullosa causing respiratory obstruction postoperatively. Anaesthesia 1991;46:422.

11. Hopkins R, Walker DM. Oral blood blisters: angina bullosa haemorrhagica. Br J Oral Maxillofac Surg 1985;23:9-16.

12. Yamamoto K, Fujimoto M, Inoue M, Maeda M, Yamakawa N, Kirita T. Angina bullosa hemorrhagica of the soft palate: report of 11 cases and literature review. J Oral Maxillofac Surg 2006;64:1433-6.

13. Giuliani M, Favia GF, Lajolo C, Miani CM. Angina bullosa haemorrhagica: presentation of eight new cases and a review of the literature. Oral Dis 2002;8:54-8.

14. Horie N, Kawano R, Inaba J, Numa T, Kato T, Nasu D, Kaneko T, Kudo I, Shimoyama T. Angina bullosa hemorrhagica of the soft palate: a clinical study of 16 cases. J Oral Sci 2008;50:33-6.

15. Corson MA, Sloan P. Angina bullosa haemorrhagica: an unusual complication following crown preparation. $\mathrm{Br}$ Dent $\mathrm{J}$ 1996;180:24-5.

16. Curran AE, Rives RW. Angina bullosa hemorrhagica: an unusual problem following periodontal therapy. J Periodontol 2000;71:1770-3.

17. O'Riordan BC. Oral blood blisters in angina bullosa haemorrhagica secondary to trauma of eating and dental injection. Br Dent J 1989;166:7.

18. High AS, Main DM. Angina bullosa haemorrhagica: a complication of long-term steroid inhaler use. $\mathrm{Br}$ Dent $\mathrm{J}$ 1988;165:176-9.

19. Porter K, Porter SR, Scully C. Angina bullosa haemorrhagica: a complication of long-term steroid inhaler use. $\mathrm{Br}$ Dent $\mathrm{J}$ 1988; 165:279.

20. Von Arx T. Bullosa haemorrhagica oralis. Rev Mens Suisse Odontostomatol 1998;108:993-6. 